\title{
The wMel Strain of Wolbachia Reduces Transmission of Chikungunya Virus in Aedes aegypti
}

\author{
Matthew T. Aliota ${ }^{1}$, Emma C. Walker ${ }^{1}$, Alexander Uribe Yepes ${ }^{2}$, Ivan Dario Velez ${ }^{2}$, Bruce \\ M. Christensen ${ }^{1}$, Jorge E. Osorio \\ 1 Department of Pathobiological Sciences, University of Wisconsin-Madison, Madison, Wisconsin, United \\ States of America, 2 Programa de Estudio y Control de Enfermedades Tropicales (PECET), Universidad de \\ Antioquia, Medellin, Colombia \\ *mtaliota@wisc.edu
}

\section{Abstract}

\section{Background}

New approaches to preventing chikungunya virus (CHIKV) are needed because current methods are limited to controlling mosquito populations, and they have not prevented the invasion of this virus into new locales, nor have they been sufficient to control the virus upon arrival. A promising candidate for arbovirus control and prevention relies on the introduction of the intracellular bacterium Wolbachia into Aedes aegypti mosquitoes. This primarily has been proposed as a tool to control dengue virus (DENV) transmission; however, evidence suggests Wolbachia infections confer protection for Ae. aegypti against CHIKV. Although this approach holds much promise for limiting virus transmission, at present our understanding of the ability of CHIKV to infect, disseminate, and be transmitted by wMel-infected $A e$. aegypti currently being used at Wolbachia release sites is limited. Agricultural Experiment Station, UNITED STATES

Received: February 25, 2016

Accepted: April 8, 2016

Published: April 28, 2016

Copyright: @ 2016 Aliota et al. This is an open access article distributed under the terms of the Creative Commons Attribution License, which permits unrestricted use, distribution, and reproduction in any medium, provided the original author and source are credited.

Data Availability Statement: All relevant data are within the paper.

Funding: This project was funded by National Institutes of Health (NIH) grant \#Al117413-01 (to JEO and MTA). The funders had no role in study design, data collection and analysis, decision to publish, or preparation of the manuscript.

Competing Interests: The authors have declared that no competing interests exist.

\section{Methodology/Principal Findings}

Using Ae. aegypti infected with the wMel strain of Wolbachia that are being released in Medellin, Colombia, we report that these mosquitoes have reduced vector competence for CHIKV, even with extremely high viral titers in the bloodmeal. In addition, we examined the dynamics of CHIKV infection over the course of four to seven days post feeding. Wolbachia-infected mosquitoes remained non-infective over the duration of seven days, i.e., no infectious virus was detected in the saliva when exposed to bloodmeals of moderate viremia, but CHIKVexposed, wild type mosquitoes did have viral loads in the saliva consistent with what has been reported elsewhere. Finally, the presence of $w$ Mel infection had no impact on the lifespan of mosquitoes as compared to wild type mosquitoes following CHIKV infection.

\section{Conclusions/Significance}

These results could have an impact on vector control strategies in areas where Ae. aegypti are transmitting both DENV and CHIKV; i.e., they argue for further exploration, both in the laboratory and the field, on the feasibility of expanding this technology beyond DENV. 


\section{Author Summary}

New approaches to preventing chikungunya virus (CHIKV) infection are needed because the endemic range of this virus is expanding and because current methods are limited to controlling mosquito populations, and this approach has not effectively controlled this virus. A promising candidate for arbovirus control and prevention relies on the introduction of the intracellular bacterium Wolbachia into Aedes aegypti mosquitoes. Wolbachia biocontrol has advanced from laboratory experiments demonstrating that Wolbachia reduces virus replication to small-scale field trials demonstrating that Wolbachia are capable of spreading through wild Ae. aegypti populations. This primarily has been proposed as a tool to control dengue virus (DENV) transmission; however, Wolbachia infections confer protection for their insect hosts against a range of pathogens including CHIKV in Ae. aegypti. Medium-scale Wolbachia deployments are imminent or in certain instances have commenced. Therefore, assessing whether or not Wolbachia-infected Ae. aegypti are effective against CHIKV will help inform the viability of Wolbachia biocontrol for CHIKV control. Our study provides valuable evidence that could justify expanding this type of control program to other Ae. aegypti-transmitted arboviruses, primarily CHIKV.

\section{Introduction}

Chikungunya virus (CHIKV; Togaviridae, Alphavirus) has recently re-emerged out of Africa and caused explosive outbreaks of arthritic disease in Southeast Asia, India, Europe and currently the Americas [1-4]. The current outbreak in the Americas is cause for great concern because $\mathrm{CHIKV}$ is spreading nearly uncontrolled with at least 44 countries experiencing autochthonous spread [5]. Infection with CHIKV results in a severe febrile illness, called chikungunya fever. Clinically, it resembles dengue fever and several other arboviral diseases [6], but it is more associated with joint pain, which in some patients can progress to chronic arthralgia that lasts for months to years [7]. CHIKV disease can be highly debilitating and has a pronounced economic impact on both the affected individual and the countries which experience the outbreaks, resulting in great losses in productivity [8-10]. CHIKV is transmitted to humans by the mosquitoes Aedes aegypti and Aedes albopictus. The distribution of these mosquitoes explains the recent global spread of the virus and invasion of the Americas [4,5,11]. Both mosquito species have demonstrated the capacity to sustain CHIKV transmission cycles and both have been associated with CHIKV outbreaks [1]; however, the etiologic strain of CHIKV, a member of the old Asian lineage [12], causing the current outbreak does not efficiently infect Ae. albopictus, suggesting that most CHIKV transmission in the Americas will occur via Ae. aegypti [5].

Despite the continued spread of the virus, there remains no effective antiviral therapy or licensed vaccines. Therefore, new approaches to preventing CHIKV are needed because the endemic range of this virus is expanding and because current methods are limited to controlling mosquito populations. To date, mosquito control has not prevented invasion of this virus into new locales or controlled the virus when it arrives [13]. A promising candidate for arbovirus control and prevention relies on the introduction of the intracellular bacterium Wolbachia into Ae. aegypti mosquitoes. Wolbachia biocontrol has advanced from laboratory experiments demonstrating that certain strains of Wolbachia shorten the lifespan of the mosquito [14] while simultaneously reducing virus replication [15] to small-scale field trials demonstrating that Wolbachia are capable of spreading through wild Ae. aegypti populations [16-18]. This primarily has been proposed as a tool to control dengue virus (DENV) transmission [19-21]; however, Wolbachia infections confer protection for their insect hosts against a range of pathogens including for $A e$. 
aegypti against CHIKV [22,23] and for Ae. albopictus against CHIKV [24]. As a result, this technology currently is being evaluated in five countries around the globe (Australia, Brazil, Colombia, Indonesia, and Vietnam) for its potential to control DENV transmission.

The approach is well-established that Wolbachia infection confers protection against DENV transmission by Ae. aegypti. In contrast, the ability of CHIKV to infect, disseminate, and be transmitted by $w \mathrm{Mel}$-infected Ae. aegypti is far less established [23]. For example, van den Hurk et al. (2012) tested the $w$ Mel strain of Wolbachia, but they only assayed Ae. aegypti vector competence for CHIKV at a single time point (12 days post feeding) with a single bloodmeal titer, and only could detect virus in the saliva via qRT-PCR [23]. And Moreira et al. (2009) tested the wMelPop strain of Wolbachia against CHIKV [22], which no longer is being utilized by the Eliminate Dengue Program (EDP) because mosquitoes infected with this strain of Wolbachia displayed reduced fitness in small-scale field releases [18]. Therefore, we assessed vector competence for CHIKV in $w$ Mel-infected and $w \mathrm{Mel}$-free Ae. aegypti from Medellin, Colombia, because at present our understanding of the ability of CHIKV to infect, disseminate, and be transmitted by $w$ Mel-infected Ae. aegypti currently being used at Wolbachia release sites is limited. This becomes particularly important if one considers that vector competence of Ae. aegypti for certain viruses likely is governed to a large extent by vector genotype $\mathrm{x}$ virus genotype $(\mathrm{G} \times \mathrm{G})$ interactions in genetically diverse, natural Ae aegypti populations [25]. This challenges the general relevance of conclusions from laboratory systems that consist of a single combination of mosquito and virus genotypes $[25,26]$. These Wolbachia-infected mosquitoes were created as part of a collaboration with the EDP in Colombia and in the spring of last year (2015), medium-scale deployments of these mosquitoes began in the DENV metropolitan area of Medellin [see www. eliminatedengue.com/colombia]. Our results suggest that Wolbachia effectively blocks the transmission potential of Colombian Ae. aegypti for CHIKV and wMel infection has no impact on the lifespan of mosquitoes as compared to wild type mosquitoes following CHIKV infection. To our knowledge, this is the first description of the effects of naturally acquired CHIKV infection (i.e., exposure to virus was accomplished by feeding on a viremic host) on Wolbachia-infected mosquito vector competence. All previous studies (including those mentioned for CHIKV, as well as the numerous studies described with DENV) have relied on animal blood spiked with cultured virus or have relied on viremic human blood from a membrane feeder. These data argue for the expansion of this technology to CHIKV in South America and are useful and germane in the broader context of CHIKV-mosquito interactions. Additionally, knowledge about factors shaping vectorial capacity (e.g., probability of daily survival) will be informative for a more accurate appraisal of CHIKV transmission and the likelihood of establishing Wolbachia infection in natural mosquito populations.

\section{Methods}

\section{Ethics statement}

This study was carried out in strict accordance with recommendations set forth in the National Institutes of Health Guide for the Care and Use of Laboratory Animals. All animals and animal facilities were under the control of the School of Veterinary Medicine with oversight from the University of Wisconsin Research Animal Resource Center. The protocol was approved by the University of Wisconsin Animal Care and Use Committee (Approval \#V01380).

\section{Cells and viruses}

African Green Monkey kidney cells (Vero; ATCC \#CCL-81) were grown in Dulbecco's modified Eagle medium (DMEM) supplemented with 10\% fetal bovine serum (FBS; Hyclone, Logan, UT), $2 \mathrm{mM} \mathrm{L}$-glutamine, $1.5 \mathrm{~g} / \mathrm{l}$ sodium bicarbonate, $100 \mathrm{U} / \mathrm{ml}$ of penicillin, $100 \mu \mathrm{g} / \mathrm{ml}$ 
of streptomycin, and incubated at $37^{\circ} \mathrm{C}$ in $5 \% \mathrm{CO}_{2}$. Aedes albopictus mosquito cells, $(\mathrm{C} 6 / 36$; ATCC \#CRL-1660) were maintained in MEM supplemented with 10\% FBS, 2 mM L-glutamine, $1.5 \mathrm{~g} / \mathrm{l}$ sodium bicarbonate, $0.1 \mathrm{mM}$ non-essential amino acids, $100 \mathrm{U} / \mathrm{ml}$ of penicillin, $100 \mu \mathrm{g} / \mathrm{ml}$ of streptomycin, and incubated at $28^{\circ} \mathrm{C}$ in $5 \% \mathrm{CO}_{2}$. CHIKV isolate 99659 (GenBank:KJ451624), originally isolated from a 33 year old male in the British Virgin Islands with a single round of amplification on Vero cells, was obtained from Brandy Russell (Centers for Disease Control and Prevention, Ft. Collins, CO, USA). Virus stocks were prepared by inoculation onto a confluent monolayer of $\mathrm{C} 6 / 36$ mosquito cells. This CHIKV strain is related phylogenetically to strains recently identified in Asia with most of them sharing a specific four amino-acid deletion in the nsP3 gene [3], and is representative of CHIKV strains circulating in Colombia [27].

\section{Mosquito strains and colony maintenance}

Ae. aegypti used in this study were maintained at the University of Wisconsin-Madison as previously described [26]. Two populations of mosquitoes were used in this study. Wild type (WT) mosquitoes (not infected with Wolbachia) were established from eggs collected from ovitraps placed around the municipality of Bello, a northwest suburb of Medellin, Colombia. The Wolbachia-infected (wMelCOL; infected with the wMel strain of Wolbachia pipientis) mosquito line was created by crossing uninfected field strains with a $w$ Mel-infected laboratory strain of Ae. aegypti essentially as described in [27]. The $w$ Mel-infected laboratory population of Ae. aegypti originated from eggs provided by Scott O'Neill (Monash University, Victoria Australia). Wolbachia infection status was routinely verified using PCR with primers specific to the IS5 repeat element [19].

\section{Exposure to infective bloodmeal}

Mosquitoes were exposed to CHIKV by feeding on isoflurane anesthetized CHIKV-infected Ifnar-/- mice. These mice have abrogated type I interferon signaling and as a result develop lethal infection, with muscle, joint, and skin serving as primary sites of replication $[28,29]$; as well, as developing high viremia. Ifnar-/- mice on the C57BL/6 background were obtained from Eva Harris (University California-Berkeley, Berkeley, CA) and were bred in the pathogen-free animal facilities of the University of Wisconsin-Madison School of Veterinary Medicine. Groups of three and six week-old mixed sex mice were used for mosquito exposures because viremia varied with age. Mice were infected in the left, hind foot pad with either $10^{3}$ plaque forming units (PFU) of CHIKV in $50 \mu \mathrm{l}$ of animal diluent (AD: $1 \%$ heat-inactivated FBS in Dulbecco's PBS) for three week-old mice or $10^{4.5} \mathrm{PFU}$ of CHIKV in $50 \mu \mathrm{l}$ of AD for six week-old mice. Uninfected mosquitoes (both $\mathrm{WT}$ and $w \mathrm{MelCOL}$ ) were allowed to feed on mice two days post infection at which time sub-mandibular blood draws were performed and serum was collected to verify viremia. Three week-old mice yielded an infectious bloodmeal concentration of $9.51 \log _{10} \mathrm{PFU} / \mathrm{ml} \pm 0.09$ (mean \pm standard deviation; $\mathrm{n}=6$ ) and six week old mice yielded an infectious bloodmeal concentration of $6.90 \log _{10} \mathrm{PFU} / \mathrm{ml} \pm 0.14$. These bloodmeal titers were consistent with human viremias observed in the field [30-32].

\section{Vector competence}

Infection, dissemination, and transmission rates were determined using long established procedures $[33,34]$. Briefly, three- to six-day-old female mosquitoes were sucrose starved for 14 to 16 hours prior to exposure to mice. Mosquitoes that fed to repletion were separated into cartons and maintained on $0.3 \mathrm{M}$ sucrose in an environmental chamber at $26.5^{\circ} \mathrm{C} \pm 1{ }^{\circ} \mathrm{C}, 75 \% \pm$ $5 \%$ relative humidity, and with a 12 hour photoperiod within the Department of Pathobiological Sciences BSL3 Insectary facility at the University of Wisconsin-Madison. All samples were 
screened by plaque assay on Vero cells. Dissemination was indicated by virus-positive legs. Transmission was defined as release of infectious virus with salivary secretions, i.e., the potential to infect another host, and was indicated by virus positive-salivary secretions.

\section{Plaque assay}

All CHIKV screens and titrations for virus quantification were completed by plaque assay on Vero cell cultures. Duplicate wells were infected with $0.1 \mathrm{ml}$ aliquots from serial 10-fold dilutions in growth media and virus was adsorbed for one hour. Following incubation, the inoculum was removed, and monolayers were overlaid with $3 \mathrm{ml}$ containing a 1:1 mixture of $1.2 \%$ oxoid agar and 2X DMEM (Gibco, Carlsbad, CA) with 10\% (vol/vol) FBS and 2\% (vol/vol) penicillin/streptomycin. Cells were incubated at $37^{\circ} \mathrm{C}$ in $5 \% \mathrm{CO}_{2}$ for two days for plaque development. Cell monolayers then were stained with $3 \mathrm{ml}$ of overlay containing a 1:1 mixture of $1.2 \%$ oxoid agar and $2 \mathrm{X}$ DMEM with $2 \%$ (vol/vol) FBS, $2 \%$ (vol/vol) penicillin/streptomycin, and $0.33 \%$ neutral red (Gibco). Cells were incubated overnight at $37^{\circ} \mathrm{C}$ and plaques were counted.

\section{Statistical analysis}

Infection, dissemination, and transmission rates were analyzed using an Exact unconditional test [35]. Saliva CHIKV titers were analyzed using a Bootstrap t-test and survival data were analyzed using Kaplan-Meir analysis and log-rank statistics.

\section{Results and Discussion}

\section{Wolbachia influences the mosquitoes' permissiveness to CHIKV infection}

In Colombia, all four DENV serotypes actively circulate in many parts of the country and there has been a significant increase in the number of severe dengue cases since re-emergence [36]. The rise in cases coincided with an increase in Ae. aegypti populations that also have expanded into new geographic locales. Similar to the country as a whole, Medellin, the second largest city in the country, also had a significant increase in dengue cases, despite the presence of a national integrated vector control strategy. This provided the impetus for new approaches to preventing DENV transmission. In fact, deployment of Wolbachia-infected Ae. aegypti began in Medellin early last year (2015) to assess the efficacy of this technology in reducing DENV transmission in endemic populations. Not surprisingly, CHIKV reached Colombia in August 2014 [25], and since its introduction, there have been over 300,000 cases of CHIKV detected. Again, current vector control measures were insufficient in preventing invasion of this virus into the country or controlling it after invasion. Although primarily designed as a biocontrol tool for DENV, evidence suggests that Wolbachia can limit infection in Ae. aegypti with CHIKV [23]; therefore, Wolbachia-infected Ae. aegypti could potentially be used to simultaneously control DENV and CHIKV. As a result, we evaluated whether Colombian mosquitoes infected with the $w \mathrm{Mel}$ strain of Wolbachia reduced CHIKV transmission potential.

Here, we verified that the phenotype of reduced vector competence existed in Wolbachiainfected laboratory colonies of Colombian Ae. aegypti for CHIKV. Adult, female, mosquitoes were exposed to infectious bloodmeals containing CHIKV and mosquitoes that ingested blood containing virus were assayed for infection, dissemination, and transmission potential at 7 and 14 days (d) post feeding (PF). As expected, infection, dissemination, and transmission rates were high for WT exposed to blood containing CHIKV at a concentration of $9.51 \log _{10} \mathrm{PFU} /$ $\mathrm{ml}$ (Table 1). Although viral titer in the bloodmeal was high, CHIKV viremia in humans can 
Table 1. Vector competence of Colombian mosquitoes following peroral infection.*

\begin{tabular}{|c|c|c|c|c|c|c|}
\hline \multicolumn{7}{|l|}{ CHIKV } \\
\hline \multirow[b]{2}{*}{ Mosquito } & \multicolumn{3}{|l|}{ 7d PF } & \multicolumn{3}{|l|}{$14 d$ PF } \\
\hline & I & D & $\mathbf{T}$ & I & D & $\mathbf{T}$ \\
\hline WT & $97(n=30)$ & $100(n=29)$ & $55(n=29)$ & $100(n=31)$ & $100(n=31)$ & $61(n=31)$ \\
\hline$w \mathrm{MeICOL}$ & $37(n=30)$ & $45(n=11)$ & 0 & $19(n=26)$ & $20(n=5)$ & $20(n=5)$ \\
\hline$p$ value $^{\dagger}$ & 0.0001 & 0.0001 & 0.0008 & 0.0001 & 0.0001 & 0.0997 \\
\hline
\end{tabular}

*I, \% Infected; D, \% Disseminated (of infected); T, \% Transmitting (of infected); bloodmeal titer $=9.51 \log _{10}$ PFU $/ \mathrm{ml}$ of CHIKV.

${ }^{\dagger}$ Calculated using an Exact unconditional test

doi:10.1371/journal.pntd.0004677.t001

vary drastically (ranging from $10^{1}-10^{9} \mathrm{PFU} / \mathrm{ml}$ ), and therefore was consistent with observations in the field [30-32]. Furthermore, evidence suggested that infection and dissemination rates were dose-dependent and rates increase with the titer of the ingested bloodmeal (see [37] for review). Our first goal then was to determine if there was a threshold in which a high viral infectious dose could overwhelm the system and negate the protection conferred by Wolbachia. Interestingly, there was a significant reduction (Exact Unconditional Test) in infection, dissemination, and transmission rates for $w$ MelCOL exposed to blood containing CHIKV; i.e., Wolbachia infection in Colombian Ae. aegypti completely blocked CHIKV transmission at $7 \mathrm{~d} P F$ and significantly reduced infection and dissemination rates at $14 \mathrm{~d} \mathrm{PF}$ (Table 1). These data were consistent with other strains of $w$ Mel-infected Ae. aegypti when exposed to CHIKV [23] or DENV [21,38]; i.e., Wolbachia infection does not completely ablate transmission of virus, but rather delays the extrinsic incubation period (EIP) of the virus and reduces the transmission potential of CHIKV-infected mosquitoes.

\section{Dynamics of infective mosquitoes}

Recently, Ye et al. (2015) demonstrated that Wolbachia-infected mosquitoes exhibited fewer infective days compared to WT mosquitoes, and their data suggested that Wolbachia-infected mosquitoes were infective at earlier timepoints [38]; however, they relied on qRT-PCR to detect and quantify virus, which does not differentiate infectious from non-infectious virus [39]. The plaque assays used here quantified infectious particles. Furthermore, it also has been demonstrated that this strain of CHIKV could be detected in the saliva of Ae. aegypti as early as $3 \mathrm{~d} \mathrm{PF}$, albeit at very low levels [40]. To ascertain if $w \mathrm{MelCOL}$ had the potential to transmit $\mathrm{CHIKV}$ at earlier time points, we assessed the dynamics of infection in WT and $w \mathrm{MelCOL}$ over time following an infectious bloodmeal more in agreement with viremias detected in Colombian patients $\left(6.90 \log _{10} \mathrm{PFU} / \mathrm{ml}\right)[25]$ versus a high viremic infectious bloodmeal $(>9.0$ $\log _{10} \mathrm{PFU} / \mathrm{ml}$ ). After a CHIKV-infected bloodmeal of moderate viremia, WT mosquitoes quickly became infective (Fig $1 \mathrm{~A}-1 \mathrm{C}$ ) and peaked at 53\% infective (10/19) at $5 \mathrm{~d}$ PF (Fig 1C). In contrast, $w$ MelCOL remained non-infective over the duration of seven days (Fig 1C), but a large proportion (39\%-70\%) of $w \mathrm{MelCOL}$ had established infections (Fig 1A) and a moderate number (11\%-29\%) also disseminated virus (Fig 1B). Likewise, after a CHIKV-infected bloodmeal of high viremia, WT mosquitoes quickly became infective (Fig 2A-2C) and maintained infectivity (Fig 2C). In contrast, $w$ MelCOL remained non-infective over the duration of seven days (Fig 2C), with the exception of a single mosquito with CHIKV-positive saliva on day six. A large proportion (up to $95 \%$ at $4 \mathrm{~d} \mathrm{PF}$ ) of $w \mathrm{MelCOL}$ had established infections (Fig 2A) and a moderate number (21-70\%) also disseminated virus (Fig 2B). Infectious virus also was detected in the saliva of $w \mathrm{MelCOL}$ on day $14 \mathrm{PF}$ (Table 1). WT mosquitoes exposed to a bloodmeal of 

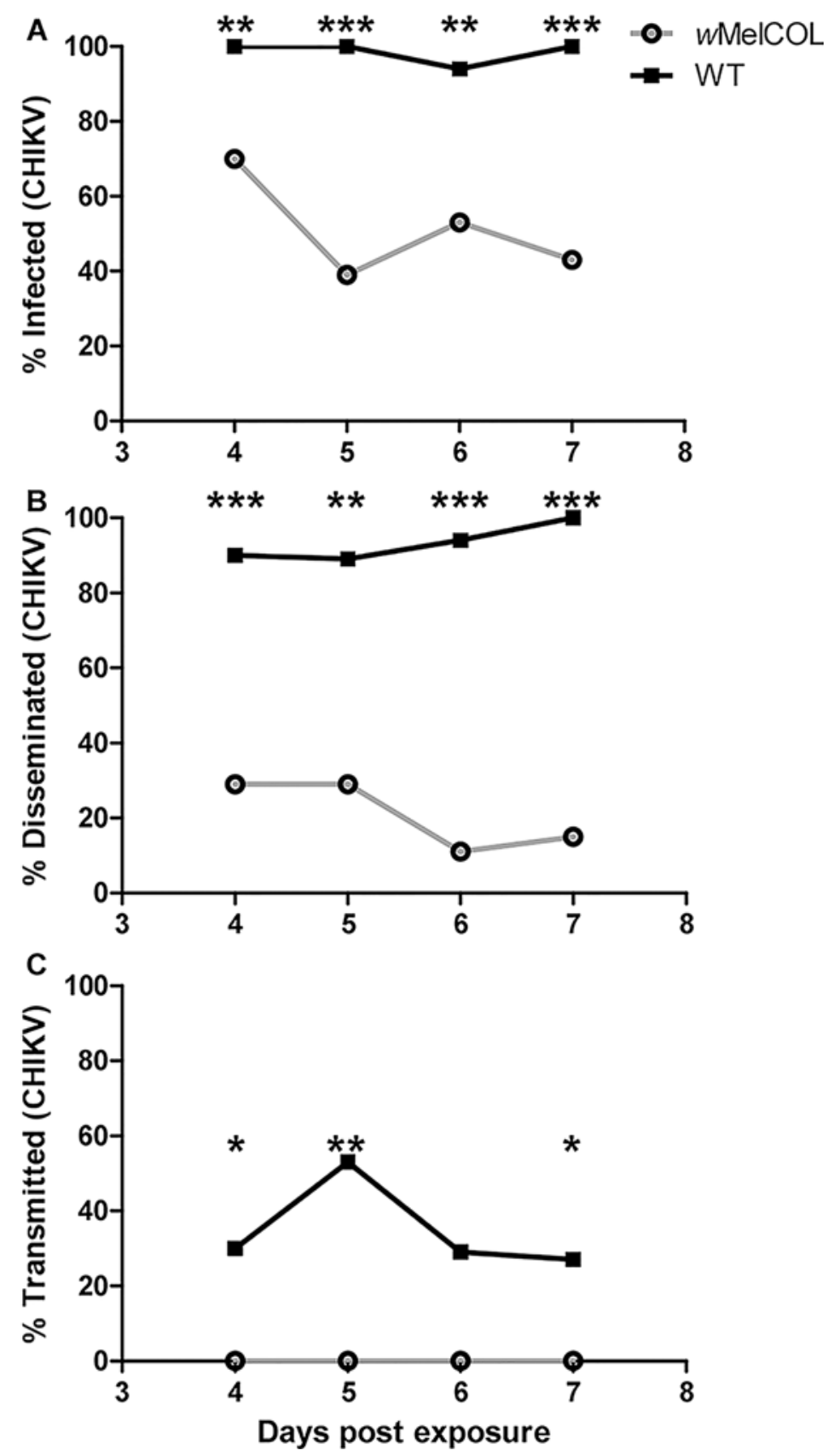

Fig 1. Infection dynamics through time for WT (black line) and wMeICOL mosquitoes (gray line) orally infected with $6.90 \log _{10}$ PFU/ml of CHIKV. Mosquitoes were examined at days 4-7 to determine infection, dissemination, and transmission efficiencies. Infection efficiency corresponds to the proportion of mosquitoes with virus-infected bodies among the tested ones. Dissemination efficiency corresponds to the proportion of mosquitoes with virus-infected legs, and transmission efficiency corresponds to the proportion of mosquitoes with infectious saliva among those infected. *, significant reduction in infection rates $\left({ }^{*} p<0.05\right.$, ${ }^{*} p<0.01$, $* * *<0.001)$. A). Percent infected ( $4 d, n=20$ for $w$ MelCOL and WT; $5 d, n=18$ for $w$ MelCOI and $n=19$ for WT; $6 \mathrm{~d}, \mathrm{n}=17$ for $w$ MelCOL and $\mathrm{n}=18$ for WT; $7 \mathrm{~d} \mathrm{n}=30$ for $w$ MelCOL and WT. B). Percent disseminated (of infected). C.) Percent transmitting (of infected).

doi:10.1371/journal.pntd.0004677.g001

high viremia had viral titers in the saliva consistent with WT exposed to a bloodmeal of moderate viremia (Fig 3). 

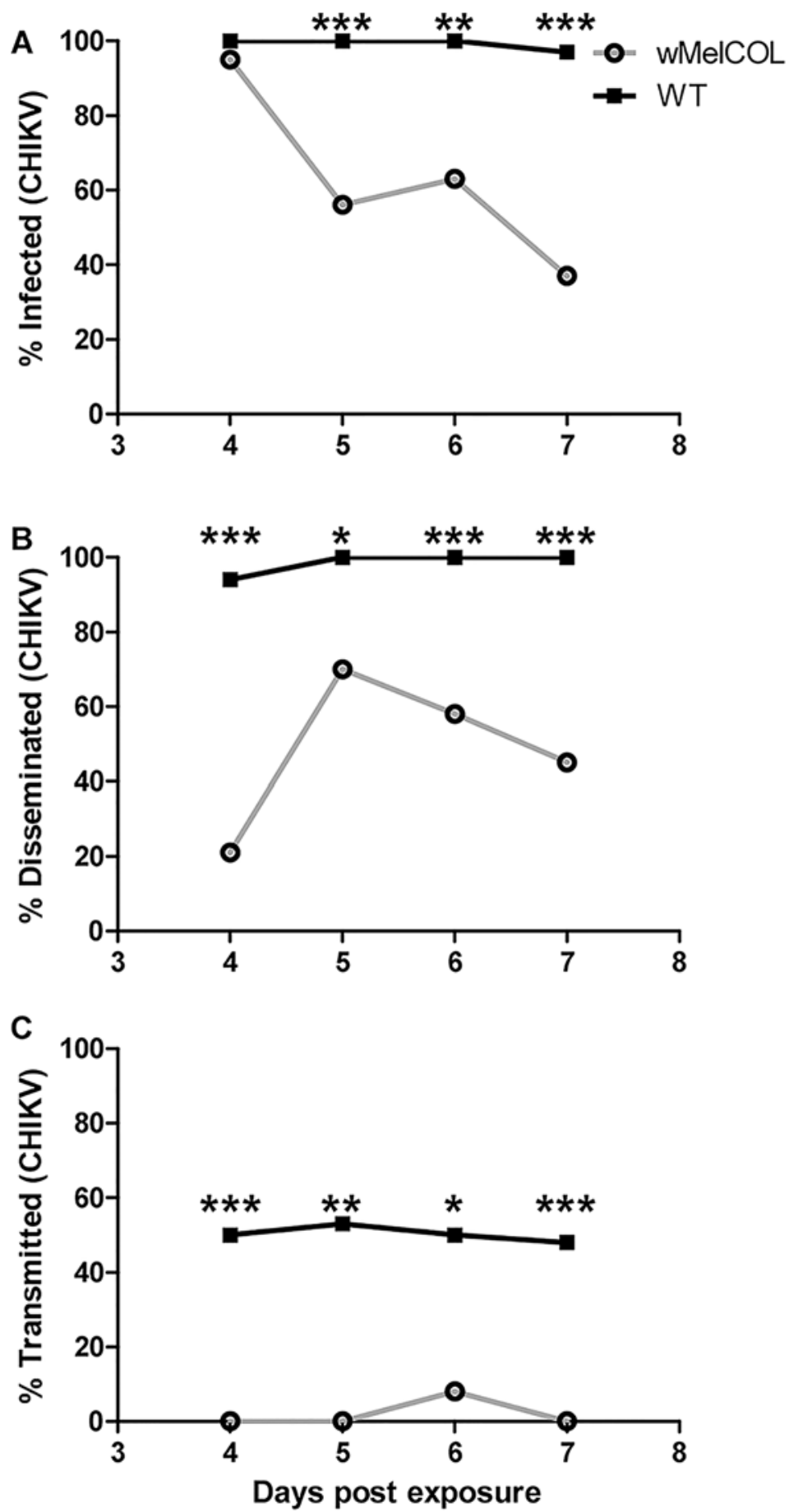

Fig 2. Infection dynamics through time for WT (black line) and wMelCOL mosquitoes (gray line) orally infected with $9.00 \log _{10} \mathrm{PFU} / \mathrm{ml}$ of CHIKV. Mosquitoes were examined at days $4-7$ to determine infection, dissemination, and transmission efficiencies. Infection efficiency corresponds to the proportion of mosquitoes with virus-infected bodies among the tested ones. Dissemination efficiency corresponds to the proportion of mosquitoes with virus-infected legs, and transmission efficiency corresponds to the proportion of mosquitoes with infectious saliva among those infected. ${ }^{*}$, significant reduction in infection rates $\left({ }^{*} p<0.05,{ }^{*} p<0.01\right.$, $* * * p<0.001)$. A). Percent infected ( $4 d, n=20$ for $w$ MelCOL and $n=18$ for WT; $5 d, n=18$ for $w$ MelCOI and $\mathrm{n}=19$ for WT; $6 \mathrm{~d}, \mathrm{n}=19$ for $w$ MelCOL and $\mathrm{n}=14$ for WT; $7 \mathrm{~d} n=30$ for $w$ MelCOL and WT. B). Percent disseminated (of infected). C.) Percent transmitting (of infected).

doi:10.1371/journal.pntd.0004677.g002 


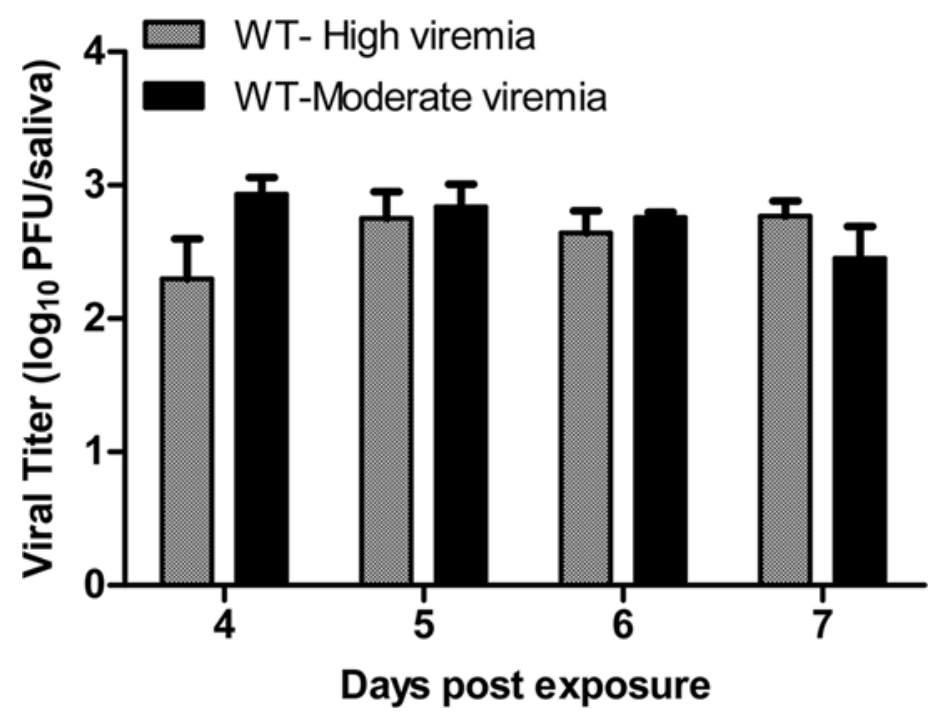

Fig 3. Viral titers in saliva of WT mosquitoes at different days after peroral infection with 6.90 or 9.00 $\log _{10}$ PFU/ml of CHIKV. Error bars represent the Bootstrap 95\% confidence interval for the mean.

doi:10.1371/journal.pntd.0004677.g003

\section{Mosquito survival post chikungunya virus infection}

We then investigated whether CHIKV had a negative effect on mosquito survival, because probability of daily survival is an important parameter in estimating vectorial capacity. It is critically important to understand how virus infection impacts vector survival if accurate predictions of transmission dynamics are to be made, because low mosquito survival will reduce the likelihood of onward transmission of the infecting virus to a new host. There has been inconsistency among reports of the effects of arboviruses on mosquito survival, and to our knowledge no reports on the impact of CHIKV infection on mosquito survival. A recent metaanalysis involving various vector-virus combinations found that, overall, arboviruses do reduce the survival of their mosquito vectors [41]. And, others have suggested that the presence of $w$ Mel infection can lengthen the lifespan of mosquitoes as compared to WT following DENV infection, suggesting that DENV infection is costly to mosquitoes and that Wolbachia is conferring some protection to the host [38]. Here, the presence of $w$ Mel infection had no impact on the lifespan of mosquitoes as compared to WT following CHIKV infection ( $p=0.369$ and $p=0.429$; Fig $4 \mathrm{~A}$ and $4 \mathrm{~B}$, respectively), nor was there any indication that CHIKV infection was overly costly to WT mosquitoes (Fig 4B). Certainly, mosquitoes survived the relatively short EIP of CHIKV (Figs 1 and 2). It also is important to note that we explored the effects of naturally acquired CHIKV infection (i.e., exposure to virus was accomplished by feeding on a viremic host) on mosquito survival; whereas, most previous studies have relied on animal blood spiked with cultured virus, which may or may not have influenced the magnitude of the observed effect. Furthermore, recent studies suggested that viral titer in the bloodmeal might impact mosquito survival; i.e., high viral titers in the blood lead to increased mosquito mortality [42]. Here, unusually high mortality was not observed in mosquitoes exposed to blood containing CHIKV at a concentration of $>9.0 \log _{10} \mathrm{PFU} / \mathrm{ml}$, i.e., a very high viral titer in the bloodmeal (Fig 4). These data are in concordance with a recent study by Carrington et al. (2015) that demonstrated that DENV infection adds minimal cost to Ae. aegypti when mosquitoes were exposed to DENV by feeding on infected humans, and there was no relationship between survival and human plasma viremia levels [43]. Although a direct comparison cannot 

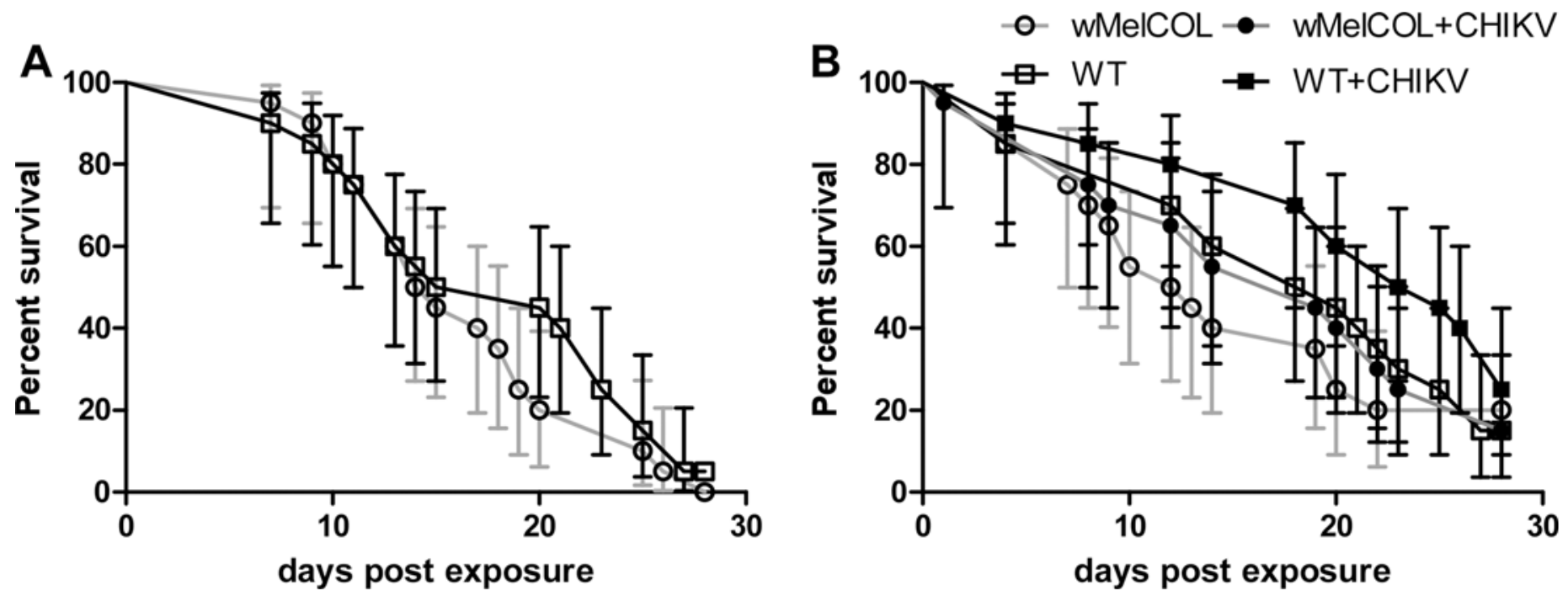

Fig 4. Survival curves of WT (black line) and wMeICOL mosquitoes (gray line) orally infected with 6.90 (A) or 9.00 (B) log ${ }_{10}$ PFU/ml of CHIKV. Error bars represent $95 \%$ confidence interval.

doi:10.1371/journal.pntd.0004677.g004

be made, our data suggest that the relationship between CHIKV and Ae. aegypti is also relatively benign; but, we cannot rule out that CHIKV and/or Wolbachia infection may impart additional costs not measured here, e.g., reduced fecundity [44]. Finally, Wolbachia biocontrol depends on Wolbachia infections being maintained stably at high levels within natural mosquito populations as well as continuing to exhibit virus interference. Wolbachia may not stably persist if there are changes in maternal transmission, cytoplasmic incompatibility, and/or fitness effects to the mosquito as a result of Wolbachia infection. Wolbachia infection did not shorten the lifespan of infected mosquitoes (Fig 4B), which bodes well for the success of this strategy, but work still is needed to assess the long-term stability of infection and changes in host fitness effects following invasion in Colombia.

In sum, Wolbachia biocontrol has been proposed primarily as a tool to control DENV transmission [19], but Wolbachia infections also confer protection for Ae. aegypti against CHIKV and to some extent yellow fever virus (YFV) [23] as well. And, as a result of the explosive outbreak of CHIKV and now Zika virus in the Western hemisphere [12,45-47], all four of these viruses co-circulate in many parts of the tropics. The possibility exists that Wolbachia biocontrol could be used as a multivalent strategy for all of these Ae. aegypti-transmitted arboviruses. At the very least, these results warrant further exploration, both in the laboratory and the field, on the feasibility of expanding this technology beyond DENV and informing whether Wolbachia biocontrol can be used to supplement or replace existing vector control strategies.

\section{Acknowledgments}

The authors thank Ryan Swanson and Jacob Patz for time spent rearing mosquitoes. We also thank the Eliminate Dengue Program for sharing Wolbachia-infected mosquitoes.

\section{Author Contributions}

Conceived and designed the experiments: MTA BMC JEO. Performed the experiments: MTA AUY ECW. Analyzed the data: MTA. Contributed reagents/materials/analysis tools: MTA IDV JEO. Wrote the paper: MTA. 


\section{References}

1. Charrel R, Leparc-Goffart I, Gallian P, de Lamballerie X. Globalization of Chikungunya: 10 years to invade the world. Clin Microbiol Infect Off Publ Eur Soc Clin Microbiol Infect Dis. 2014; doi: 10.1111/ 1469-0691.12694

2. Gould EA, Gallian P, De Lamballerie X, Charrel RN. First cases of autochthonous dengue fever and chikungunya fever in France: from bad dream to reality! Clin Microbiol Infect Off Publ Eur Soc Clin Microbiol Infect Dis. 2010; 16: 1702-1704. doi: 10.1111/j.1469-0691.2010.03386.x

3. Leparc-Goffart I, Nougairede A, Cassadou S, Prat C, de Lamballerie X. Chikungunya in the Americas. Lancet. 2014; 383: 514. doi: 10.1016/S0140-6736(14)60185-9 PMID: 24506907

4. Charrel RN, de Lamballerie $X$, Raoult $D$. Chikungunya outbreaks-the globalization of vectorborne diseases. N Engl J Med. 2007; 356: 769-771. doi: 10.1056/NEJMp078013 PMID: 17314335

5. Vega-Rúa A, Zouache K, Girod R, Failloux A-B, Lourenço-de-Oliveira R. High Level of Vector Competence of Aedes aegypti and Aedes albopictus from Ten American Countries as a Crucial Factor in the Spread of Chikungunya Virus. J Virol. 2014; 88: 6294-6306. doi: 10.1128/JVI.00370-14 PMID: 24672026

6. Thiberville S-D, Moyen N, Dupuis-Maguiraga L, Nougairede A, Gould EA, Roques P, et al. Chikungunya fever: epidemiology, clinical syndrome, pathogenesis and therapy. Antiviral Res. 2013; 99: 345370. doi: 10.1016/j.antiviral.2013.06.009 PMID: 23811281

7. Powers AM. Chikungunya. Clin Lab Med. 2010; 30: 209-219. doi: 10.1016/j.cll.2009.10.003 PMID: 20513548

8. Vijayakumar K, George B, Anish TS, Rajasi RS, Teena MJ, Sujina CM. Economic impact of chikungunya epidemic: out-of-pocket health expenditures during the 2007 outbreak in Kerala, India. Southeast Asian J Trop Med Public Health. 2013; 44: 54-61. PMID: 23682438

9. Krishnamoorthy K, Harichandrakumar KT, Krishna Kumari A, Das LK. Burden of chikungunya in India: estimates of disability adjusted life years (DALY) lost in 2006 epidemic. J Vector Borne Dis. 2009; 46: 26-35. PMID: 19326705

10. Cardona-Ospina JA, Villamil-Gómez WE, Jimenez-Canizales CE, Castañeda-Hernández DM, Rodríguez-Morales AJ. Estimating the burden of disease and the economic cost attributable to chikungunya, Colombia, 2014. Trans R Soc Trop Med Hyg. 2015; 109: 793-802. doi: 10.1093/trstmh/trv094 PMID: 26626342

11. Tsetsarkin KA, McGee CE, Volk SM, Vanlandingham DL, Weaver SC, Higgs S. Epistatic roles of E2 glycoprotein mutations in adaption of chikungunya virus to Aedes albopictus and Ae. aegypti mosquitoes. PloS One. 2009; 4: e6835. doi: 10.1371/journal.pone.0006835 PMID: 19718263

12. Lanciotti RS, Valadere AM. Transcontinental movement of Asian genotype chikungunya virus. Emerg Infect Dis. 2014; 20: 1400-1402. doi: 10.3201/eid2008.140268 PMID: 25076384

13. Maciel-de-Freitas R, Aguiar R, Bruno RV, Guimarães MC, Lourenço-de-Oliveira R, Sorgine MHF, et al. Why do we need alternative tools to control mosquito-borne diseases in Latin America? Mem Inst Oswaldo Cruz. 2012; 107: 828-829. PMID: 22990977

14. McMeniman CJ, Lane RV, Cass BN, Fong AWC, Sidhu M, Wang Y-F, et al. Stable introduction of a lifeshortening Wolbachia infection into the mosquito Aedes aegypti. Science. 2009; 323: 141-144. doi: 10 . 1126/science. 1165326 PMID: 19119237

15. Rasgon JL, Scott TW. Impact of population age structure on Wolbachia transgene driver efficacy: ecologically complex factors and release of genetically modified mosquitoes. Insect Biochem Mol Biol. 2004; 34: 707-713. doi: 10.1016/j.ibmb.2004.03.023 PMID: 15242712

16. Hoffmann AA, Montgomery BL, Popovici J, Iturbe-Ormaetxe I, Johnson PH, Muzzi F, et al. Successful establishment of Wolbachia in Aedes populations to suppress dengue transmission. Nature. 2011; 476: 454-457. doi: 10.1038/nature10356 PMID: 21866160

17. Hoffmann AA, Iturbe-Ormaetxe I, Callahan AG, Phillips BL, Billington K, Axford JK, et al. Stability of the wMel Wolbachia Infection following Invasion into Aedes aegypti Populations. PLoS Negl Trop Dis. 2014; 8: e3115. doi: 10.1371/journal.pntd.0003115 PMID: 25211492

18. Nguyen TH, Nguyen HL, Nguyen TY, Vu SN, Tran ND, Le TN, et al. Field evaluation of the establishment potential of wmelpop Wolbachia in Australia and Vietnam for dengue control. Parasit Vectors. 2015; 8: 563. doi: 10.1186/s13071-015-1174-x PMID: 26510523

19. Walker T, Johnson PH, Moreira LA, Iturbe-Ormaetxe I, Frentiu FD, McMeniman CJ, et al. The wMe Wolbachia strain blocks dengue and invades caged Aedes aegypti populations. Nature. $2011 ; 476$ : 450-453. doi: 10.1038/nature10355 PMID: 21866159

20. Hoffmann AA, Montgomery BL, Popovici J, Iturbe-Ormaetxe I, Johnson PH, Muzzi F, et al. Successful establishment of Wolbachia in Aedes populations to suppress dengue transmission. Nature. 2011; 476: 454-457. doi: 10.1038/nature10356 PMID: 21866160 
21. Frentiu FD, Zakir T, Walker T, Popovici J, Pyke AT, van den Hurk A, et al. Limited Dengue Virus Replication in Field-Collected Aedes aegypti Mosquitoes Infected with Wolbachia. PLoS Negl Trop Dis. 2014; 8: e2688. doi: 10.1371/journal.pntd.0002688 PMID: 24587459

22. Moreira LA, Iturbe-Ormaetxe I, Jeffery JA, Lu G, Pyke AT, Hedges LM, et al. A Wolbachia symbiont in Aedes aegypti limits infection with dengue, Chikungunya, and Plasmodium. Cell. 2009; 139: 12681278. doi: 10.1016/j.cell.2009.11.042 PMID: 20064373

23. van den Hurk AF, Hall-Mendelin S, Pyke AT, Frentiu FD, McElroy K, Day A, et al. Impact of Wolbachia on infection with chikungunya and yellow fever viruses in the mosquito vector Aedes aegypti. PLoS Negl Trop Dis. 2012; 6: e1892. doi: 10.1371/journal.pntd.0001892 PMID: 23133693

24. Blagrove MSC, Arias-Goeta C, Di Genua C, Failloux A-B, Sinkins SP. A Wolbachia wMel transinfection in Aedes albopictus is not detrimental to host fitness and inhibits Chikungunya virus. PLoS Negl Trop Dis. 2013; 7: e2152. doi: 10.1371/journal.pntd.0002152 PMID: 23556030

25. Mattar S, Miranda J, Pinzon H, Tique V, Bolanos A, Aponte J, et al. Outbreak of Chikungunya virus in the north Caribbean area of Colombia: clinical presentation and phylogenetic analysis. J Infect Dev Ctries. 2015; 9: 1126-1132. doi: 10.3855/jidc.6670 PMID: 26517488

26. Christensen BM, Sutherland DR. Brugia pahangi: Exsheathment and Midgut Penetration in Aedes aegypti. Trans Am Microsc Soc. 1984; 103: 423-433.

27. Yeap HL, Mee P, Walker T, Weeks AR, O'Neill SL, Johnson P, et al. Dynamics of the "popcorn" Wolbachia infection in outbred Aedes aegypti informs prospects for mosquito vector control. Genetics. 2011 187: 583-595. doi: 10.1534/genetics.110.122390 PMID: 21135075

28. Couderc T, Chrétien F, Schilte C, Disson O, Brigitte M, Guivel-Benhassine F, et al. A mouse model for Chikungunya: young age and inefficient type-I interferon signaling are risk factors for severe disease. PLoS Pathog. 2008; 4: e29. doi: 10.1371/journal.ppat.0040029 PMID: 18282093

29. Gardner CL, Burke CW, Higgs ST, Klimstra WB, Ryman KD. Interferon-alpha/beta deficiency greatly exacerbates arthritogenic disease in mice infected with wild-type chikungunya virus but not with the cell culture-adapted live-attenuated 181/25 vaccine candidate. Virology. 2012; 425: 103-112. doi: 10.1016/ j.virol.2011.12.020 PMID: 22305131

30. Thiberville S-D, Boisson V, Gaudart J, Simon F, Flahault A, de Lamballerie X. Chikungunya Fever: A Clinical and Virological Investigation of Outpatients on Reunion Island, South-West Indian Ocean. PLoS Negl Trop Dis. 2013; 7. doi: 10.1371/journal.pntd.0002004

31. Appassakij H, Khuntikij $P$, Kemapunmanus M, Wutthanarungsan R, Silpapojakul K. Viremic profiles in asymptomatic and symptomatic chikungunya fever: a blood transfusion threat? Transfusion (Paris). 2013; 53: 2567-2574. doi: 10.1111/j.1537-2995.2012.03960.x

32. Leo YS, Chow ALP, Tan LK, Lye DC, Lin L, Ng LC. Chikungunya outbreak, Singapore, 2008. Emerg Infect Dis. 2009; 15: 836-837. doi: 10.3201/eid1505.081390 PMID: 19402989

33. Aliota MT, Jones SA, Dupuis AP 2nd, Ciota AT, Hubalek Z, Kramer LD. Characterization of Rabensburg virus, a flavivirus closely related to West Nile virus of the Japanese encephalitis antigenic group. PloS One. 2012; 7: e39387. doi: 10.1371/journal.pone.0039387 PMID: 22724010

34. Weger-Lucarelli J, Aliota MT, Wlodarchak N, Kamlangdee A, Swanson R, Osorio JE. Dissecting the role of E2 protein domains on alphavirus pathogenicity. J Virol. 2015; doi: 10.1128/JVI.02792-15

35. Reiczigel J, Abonyi-Tóth Z, Singer J. An exact confidence set for two binomial proportions and exact unconditional confidence intervals for the difference and ratio of proportions. Comput Stat Data Anal. 2008; 52: 5046-5053. doi: 10.1016/j.csda.2008.04.032

36. Restrepo BN, Piedrahita LD, Agudelo IY, Parra-Henao G, Osorio JE. Frequency and clinical features of dengue infection in a schoolchildren cohort from medellin, Colombia. J Trop Med. 2012; 2012: 120496. doi: 10.1155/2012/120496 PMID: 23304167

37. Coffey LL, Failloux A-B, Weaver SC. Chikungunya Virus-Vector Interactions. Viruses. 2014; 6: 46284663. doi: 10.3390/v6114628 PMID: 25421891

38. Ye YH, Carrasco AM, Frentiu FD, Chenoweth SF, Beebe NW, van den Hurk AF, et al. Wolbachia Reduces the Transmission Potential of Dengue-Infected Aedes aegypti. PLoS Negl Trop Dis. 2015; 9: e0003894. doi: 10.1371/journal.pntd.0003894 PMID: 26115104

39. Hue KDT, Tuan TV, Thi HTN, Bich CTN, Anh HHL, Wills BA, et al. Validation of an internally controlled one-step real-time multiplex RT-PCR assay for the detection and quantitation of dengue virus RNA in plasma. J Virol Methods. 2011; 177: 168-173. doi: 10.1016/j.jviromet.2011.08.002 PMID: 21843553

40. Vega-Rúa A, Lourenço-de-Oliveira R, Mousson L, Vazeille M, Fuchs S, Yébakima A, et al. Chikungunya Virus Transmission Potential by Local Aedes Mosquitoes in the Americas and Europe. PLoS Negl Trop Dis. 2015; 9: e0003780. doi: 10.1371/journal.pntd.0003780 PMID: 25993633

41. Lambrechts $L$, Scott TW. Mode of transmission and the evolution of arbovirus virulence in mosquito vectors. Proc Biol Sci. 2009; 276: 1369-1378. doi: 10.1098/rspb.2008.1709 PMID: 19141420 
42. Maciel-de-Freitas R, Koella JC, Lourenço-de-Oliveira R. Lower survival rate, longevity and fecundity of Aedes aegypti (Diptera: Culicidae) females orally challenged with dengue virus serotype 2 . Trans $R$ Soc Trop Med Hyg. 2011; 105: 452-458. doi: 10.1016/j.trstmh.2011.05.006 PMID: 21700303

43. Carrington LB, Nguyen HL, Nguyen NM, Duong THK, Tuan TV, Giang NT, et al. Naturally-acquired dengue virus infections do not reduce short-term survival of infected Aedes aegypti from Ho Chi Minh City, Vietnam. Am J Trop Med Hyg. 2015; 92: 492-496. doi: 10.4269/ajtmh.14-0499 PMID: 25561566

44. Ciota AT, Ehrbar DJ, Matacchiero AC, Van Slyke GA, Kramer LD. The evolution of virulence of West Nile virus in a mosquito vector: implications for arbovirus adaptation and evolution. BMC Evol Biol. 2013; 13: 71. doi: 10.1186/1471-2148-13-71 PMID: 23514328

45. Musso D. Zika Virus Transmission from French Polynesia to Brazil. Emerg Infect Dis. 2015; 21: 1887. doi: 10.3201/eid2110.151125

46. Musso D, Cao-Lormeau VM, Gubler DJ. Zika virus: following the path of dengue and chikungunya? Lancet Lond Engl. 2015; 386: 243-244. doi: 10.1016/S0140-6736(15)61273-9

47. Erwin Camacho, Margaret Paternina-Gomez, Blanco Pedro J., Osorio Jorge E., Aliota Matthew T. Detection of Autochthonous Zika Virus Transmission in Sincelejo, Colombia. Emerg Infect Dis J. 2016;22. doi: 10.3201/eid2205.160023 